\title{
BMJ Global Health Impact of overweight and obesity on life expectancy, quality-adjusted life years and lifetime costs in the adult population of Ghana
}

\author{
Stella Lartey (D) , 1,2,3 Lei Si (D) , 4,5 Thomas Lung, ${ }^{4,6}$ Costan G Magnussen, ${ }^{1,7}$ \\ Godfred O Boateng, ${ }^{8}$ Nadia Minicuci, ${ }^{9}$ Paul Kowal, ${ }^{10,11}$ Alison Hayes, ${ }^{6}$ \\ Barbara de Graaff, ${ }^{1}$ Leigh Blizzard, ${ }^{1}$ Andrew J Palmer ${ }^{1,12}$
}

\begin{abstract}
To cite: Lartey S, Si L, Lung T, et al. Impact of overweight and obesity on life expectancy, quality-adjusted life years and lifetime costs in the adult population of Ghana. BMJ Global Health 2020;5:e003332. doi:10.1136/ bmjgh-2020-003332
\end{abstract}

Handling editor Sanni Yaya

- Additional material is published online only. To view please visit the journal online (http://dx.doi.org/10.1136/ bmjgh-2020-003332).

Received 4 July 2020 Revised 5 August 2020 Accepted 9 August 2020
Check for updates

(c) Author(s) (or their employer(s)) 2020. Re-use permitted under CC BY-NC. No commercial re-use. See rights and permissions. Published by BMJ.

For numbered affiliations see end of article.

Correspondence to Dr Stella Lartey; stella.lartey@utas.edu.au

\section{ABSTRACT}

Introduction Prior studies have revealed the increasing prevalence of obesity and its associated health effects among ageing adults in resource poor countries. However, no study has examined the long-term and economic impact of overweight and obesity in subSaharan Africa. Therefore, we quantified the long-term impact of overweight and obesity on life expectancy (LE), quality-adjusted life years (QALYs) and total direct healthcare costs.

Methods A Markov simulation model projected health and economic outcomes associated with three categories of body mass index (BMI): healthy weight $(18.5 \leq \mathrm{BMI}<25.0)$; overweight $(25.0 \leq \mathrm{BMI}<30.0)$ and obese $\left(B M I \geq 30.0 \mathrm{~kg} / \mathrm{m}^{2}\right.$ ) in simulated adult cohorts over a 50 -year time horizon from age fifty. Costs were estimated from government and patient perspectives, discounted 3\% annually and reported in 2017 US\$. Mortality rates from Ghanaian lifetables were adjusted by BMI-specific all-cause mortality HRs. Published input data were used from the 2014/2015 Ghana WHO Study on global AGEing and adult health data. Internal and external validity were assessed.

Results From age 50 years, average $(95 \% \mathrm{Cl})$ remaining LE for females were 25.6 (95\% Cl: 25.4 to 25.8), 23.5 (95\% Cl: 23.3 to 23.7$)$ and 21.3 (95\% Cl: 19.6 to 21.8$)$ for healthy weight, overweight and obesity, respectively. In males, remaining LE were healthy weight (23.0; 95\% Cl: 22.8 to 23.2), overweight (20.7; 95\% Cl: 20.5 to 20.9$)$ and obesity (17.6; $95 \% \mathrm{Cl}: 17.5$ to 17.8$)$. In females, QALYs for healthy weight were $23.0(95 \% \mathrm{Cl}$ : 22.8 to 23.2), overweight, 21.0 (95\% Cl: 20.8 to 21.2) and obesity, 19.0 (95\% Cl: 18.8 to 19.7). The discounted total costs per female were US\$619 (95\% Cl: 616 to 622), US\$1298 (95\% Cl: 1290 to 1306) and US\$2057 (95\% Cl: 2043 to 2071) for healthy weight, overweight and obesity, respectively. QALYS and costs were lower in males.

Conclusion Overweight and obesity have substantial health and economic impacts, hence the urgent need for cost-effective preventive strategies in the Ghanaian population.

\section{Key messages}

What is already known?

- Obesity is a significant global public health challenge because it is a major risk factor for many noncommunicable diseases (NCDs) and independently predicts overall mortality.

- While its prevalence has increased, there is very scanty information on the long-term health and economic impacts of obesity in sub-Saharan Africa.

\section{What are the new findings?}

- To our knowledge, this is the first health economic modelling study that predicts the long-term health and economic impacts of obesity in the Ghanaian adult population.

- Our results show that overweight and obesity are associated with substantial health and economic burden.

What do the new findings imply?

- As a major risk factor for NCDs, our evidence reinforces WHO's anticipated double burden of communicable and NCDs in the near future in most low-income and middle-income countries.

- The substantial economic burden found will significantly increase over the coming decades if proactive steps are not implemented to reduce the increasing prevalence of overweight and obesity.

- This study has developed a readily available model to support cost-effectiveness analysis of obesity preventive interventions.

\section{INTRODUCTION}

Obesity (body mass index, BMI $\geq 30.0 \mathrm{~kg}$ / $\mathrm{m}^{2}$ ) is one of the leading risk factors for non-communicable diseases (NCDs) and has become a major global health concern. ${ }^{1}$ Since 1980, the prevalence of obesity has doubled among adults in most parts of the 
world including sub-Saharan Africa. ${ }^{12}$ Obesity substantially increases morbidity, disability and mortality, and poses a significant economic burden to populations. ${ }^{13}$ In spite of the difficulty associated with its management, ${ }^{4}$ health consequences and cost burden, ${ }^{35}$ the prevalence of obesity is increasing in many places. ${ }^{26}$ This increase may present a major challenge to households, clinicians, health systems and, especially, decision-makers when allocating resources to prevent or manage obesity ${ }^{7}$ and obesity-related disabilities.

Interventions to prevent or manage obesity in populations with high prevalence can be resource intensive. As obesity is a major risk factor for NCDs, evaluating associated policies could pose significant challenges as NCDs impose enormous health and economic effects. ${ }^{15}$ While clinical trials are limited by time, and therefore can provide evidence of short-term to medium-term effects, economic modelling is a method that can provide estimates of the possible long-term effects. ${ }^{8}$ Estimating the long-term effects of overweight and obesity are necessary to identify differences in subpopulations, inform the development and correctly target obesity prevention and management strategies, and the allocation of health resources. ${ }^{9}$ A key long-term metric that has also been useful in communicating the impact of obesity is years of life lost (YLL) ${ }^{10}$ In this context, YLL is estimated as the difference between the remaining life expectancy (LE) of a person with healthy weight and that of person with overweight or obesity. ${ }^{9} 10$ Previous studies have shown that compared with healthy weight, the remaining LE due to obesity is lower. ${ }^{9-11}$

Despite the challenges obesity presents, ${ }^{12}$ there is a dearth of studies estimating the long-term impacts of obesity on health and economic outcomes in sub-Saharan Africa. Therefore, the aim of this study was to quantify the longterm health and economic effects of overweight and obesity in Ghana. Specifically, we reported remaining LE, YLL, quality-adjusted life years (QALYs) and the direct healthcare costs stratified by sex for healthy weight, overweight and obesity for individuals aged 50 years at baseline. Finally, we estimated the YLL, QALYs and total costs scaled up to the entire 50-year-old population with overweight and obesity, compared with those with healthy weight.

\section{METHODS}

\section{Model description}

A Markov model that used survey-estimated parameters was adapted in this study to simulate the health and costs outcomes for a hypothetical adult cohort of 10000 subjects, ${ }^{13} 14$ accounting for age, sex and BMI status. The model generated remaining LE, YLL, QALYs and costs for persons with healthy weight, overweight and obesity. Secondorder Monte Carlo simulation in which the values were simultaneously drawn from multiple distributions was performed. The cycle length was 1 year and the subjects, with initial age of 50 years, were simulated until death or until age 100 years. This study used an initial age of 50 years due to the availability of key input parameters for this age. ${ }^{15-17}$ To estimate the impact of different categories of BMI on remaining LE, YLL, QALYs and costs, simulated subjects were assumed to remain in their BMI category for their lifetime. Even though in reality there might be changes in the BMI of these individuals, there is little information to estimate the annual BMI changes for this population.

There were two health states: alive and dead with dead as an absorbing state. ${ }^{18}$ A subject either remained in the alive state or moved onto the next cycle or died (figure 1). The probability of death was determined by the mortality rates from the Ghanaian general population adjusted according to the BMI category. ${ }^{14}$ BMIspecific mortality was estimated as a function of age, sex and BMI category. Current age-specific and sex-specific prevalences of individuals in each BMI category were estimated in our previous studies, ${ }^{15}$ age-specific and sexspecific mortality rates were taken from the 2015 Ghana

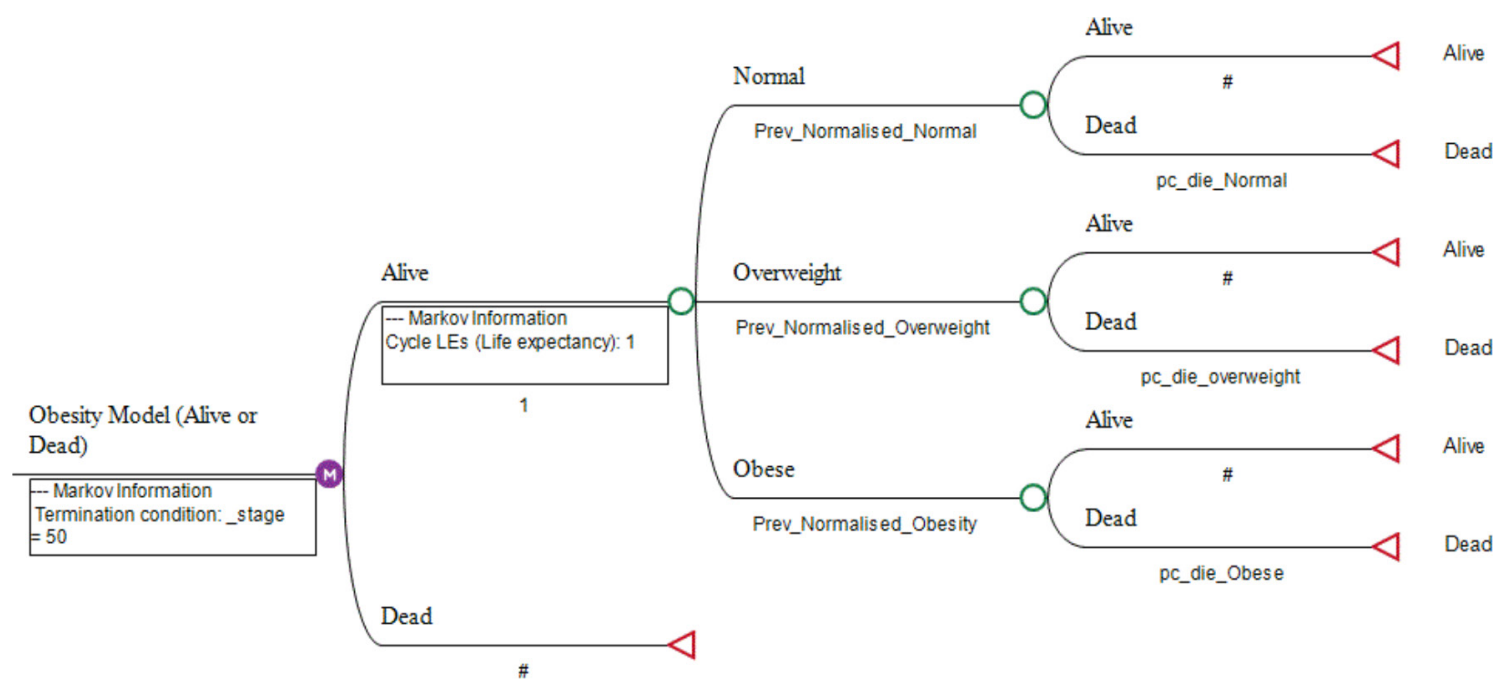

Figure 1 Structure of the Markov model. \# denotes the remainder of the probability at the terminal node. LE, life expectancies. 
Table 1 Key parameters in the model (prevalence, health state utilities and mortality rates)

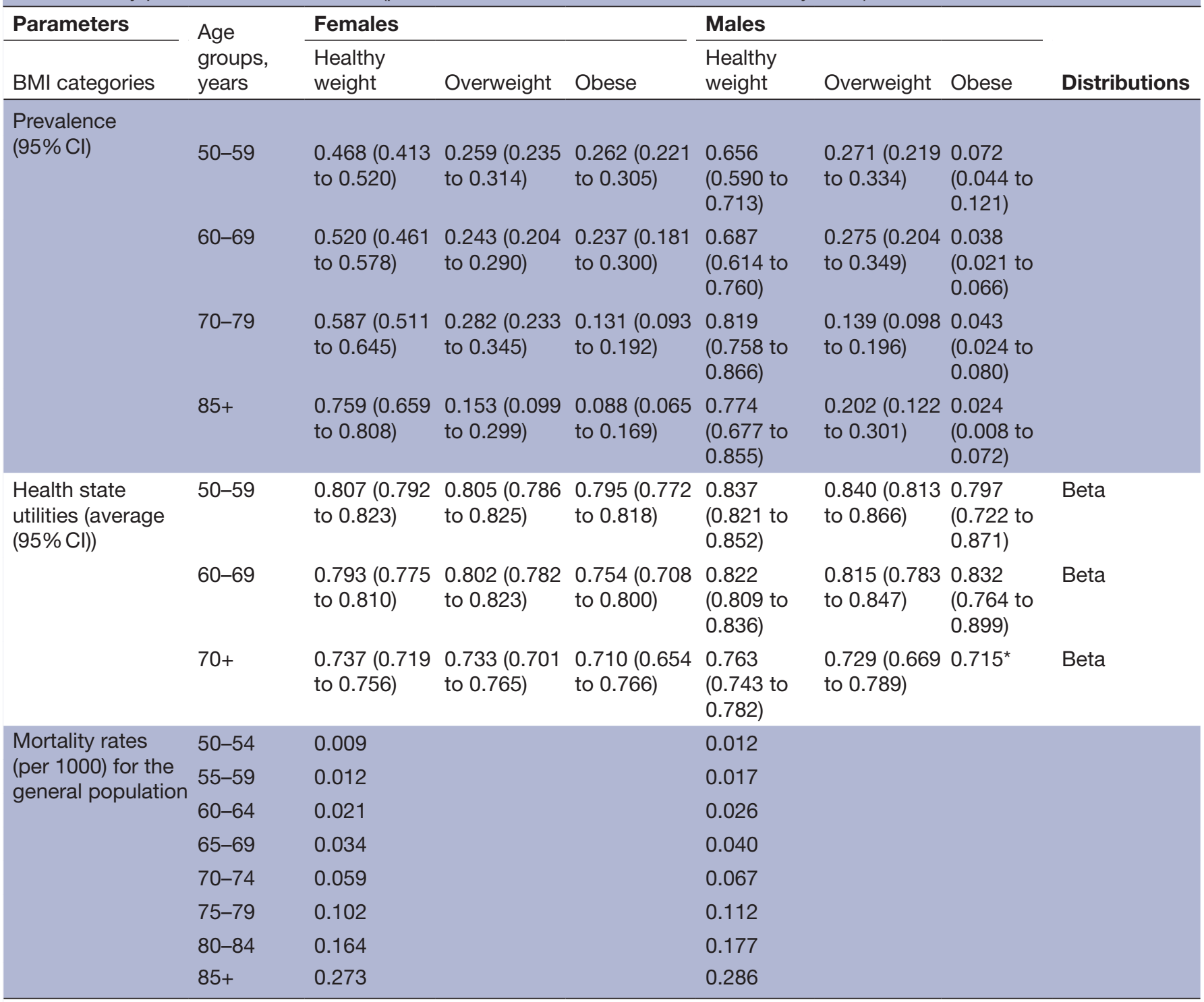

Prevalence, ${ }^{15}$ health state utilities ${ }^{16}$ and mortality rates ${ }^{19}$ were taken from previous studies.

${ }^{*} \mathrm{Cls}$ were not estimated due to insufficient data, (the subsample for males with obesity in age group 70 years and above, $n=13$ ).

$\mathrm{BMI}$, body mass index.

life tables $^{19}$ (table 1), and BMI-specific HRs of all-cause mortality taken from the published literature ${ }^{20}$ (table 2). An increased risk of mortality for each 5-unit increase of

\begin{tabular}{|c|c|c|c|}
\hline \multirow[b]{2}{*}{ *HR } & \multicolumn{2}{|l|}{ BMI ranges } & \multirow[b]{2}{*}{ Distributions } \\
\hline & $15-25 \mathrm{~kg} / \mathrm{m}^{2}$ & $25-50 \mathrm{~kg} / \mathrm{m}^{2}$ & \\
\hline \multicolumn{4}{|c|}{$\begin{array}{l}\text { Age } \\
\text { groups, } \\
\text { years }\end{array}$} \\
\hline $35-59$ & $0.76(0.71-0.81)$ & $1.37(1.31-1.42)$ & Not reported \\
\hline $60-69$ & $0.77(0.73-0.82)$ & $1.32(1.27-1.36)$ & \\
\hline $70-79$ & $0.82(0.77-0.87)$ & $1.27(1.23-1.32)$ & \\
\hline $80-89$ & $0.89(0.80-0.97)$ & $1.16(1.10-1.23)$ & \\
\hline
\end{tabular}

${ }^{*} \mathrm{HR}$ increased per $5 \mathrm{~kg} / \mathrm{m}^{2}$ unit increase of BMl. ${ }^{20}$
BMI allowed the variation of mortality for subjects based on the BMI category. ${ }^{90}$ The methods used to determine mortality rates have been described elsewhere. ${ }^{9}$

Health state utilities (HSUs) ${ }^{16}$ and direct healthcare costs data $^{17}$ for each BMI category were simulated using beta and gamma distributions, respectively. The model was constructed using TreeAge Pro Suite V.2018 R1.1 (TreeAge Software, Williamstown, Massachusetts, USA). One-way (shown in tornado diagram) and probabilistic sensitivity analyses were used to address parameter uncertainty. The model was tested on its face, internal and external validity.

\section{Study population}

Parameters including the prevalence of the BMI categories, ${ }^{15} \mathrm{HSUs}^{16}$ and direct healthcare costs ${ }^{17}$ were estimated using the 2014/2015 Ghana WHO's Study on global AGEing and adult health (WHO SAGE). The parameters 
estimated for the older adult who were 50 years and above were used for this modelling exercise as they were nationally representative in the WHO SAGE data. ${ }^{15-1721}$ Additionally, while data on prevalence and HSUs were available for all adults aged 18 years and older, costs data were available only for those above 50 years. ${ }^{15-17}$ Of the 4735 survey respondents, 1114 were excluded as they were less than 50 years of age or had missing or biologically implausible weight (weight $<30.0 \mathrm{~kg}$ or $>250.0 \mathrm{~kg}$ ), height (height $<100 \mathrm{~cm}$ or $>250 \mathrm{~cm}$ ) and waist (circumference $<25.0 \mathrm{~cm}$ or $>220 \mathrm{~cm}$ ) measurements ${ }^{22} 23$; resulting in 3350 for analysis. SAGE used a stratified multistage cluster design to collect data that yielded national and subnational estimates with acceptable precision using region and locality (rural/urban) as the primary sampling unit. ${ }^{21}$. Survey weights provided by WHO SAGE were applied in all estimations to ensure that values are representative of the Ghanaian population. Please visit http://www.who.int/ healthinfo/sage/cohorts/en for further information on the WHO SAGE.

\section{Base case population}

The WHO SAGE database was the source of most of the model's parameters. As such, the base case population was Ghanaian adults aged 50 years.

\section{BMI measurement}

In the WHO SAGE data, anthropometric measurements of body weight and height of respondents were taken by trained interviewers using a weighing scale and stadiometer following standard protocols. ${ }^{21}$ BMI was calculated as a person's weight in kilograms divided by the square of their height in metres and obesity was defined using cut-offs following the WHO classification. Three BMI $\left(\mathrm{kg} / \mathrm{m}^{2}\right)$ categories were used: healthy weight, $18.5 \leq \mathrm{BMI}$ $<25.0$; overweight, $25.0 \leq \mathrm{BMI}<30.0$; and obesity as BMI $\geq 30.0{ }^{24}$ Pregnant women were exempted from weight measurements and excluded from this analysis. ${ }^{21}$

\section{Health state utilities}

BMI category-specific HSUs were used to calculate QALYs. These were taken from a published study conducted by our group using the Ghanaian population. ${ }^{16}$ It is the only study to-date that has estimated BMI-related utility values in this population. This study reported HSUs among a nationally representative sample of Ghanaian adults, stratified by age, sex and BMI categories (table 1).

\section{Costs}

Costs were used from both the government and patient perspectives and were limited to direct healthcare costs (table 3). These costs, stratified by BMI categories were taken from our previous study. ${ }^{17}$ Costs included self-reported out-of-pocket costs and the government National Health Insurance Scheme (NHIS) claims, both of which were estimated for outpatient consultations and inpatient admissions. Total costs were estimated as the sum of out-of-pocket and the government's NHIS costs. All costs were then converted into US\$ equivalent using the 2017 average exchange rate (US $\$ 1 \approx$ GHS 4.3562). Future costs were discounted at $3 \%$.

\section{YLL, QALYs losses and extra costs}

Using healthy weight as a comparator, we estimated the average YLL, QALYs lost and the extra direct healthcare total costs due to overweight and obesity. Average YLL was estimated as the difference in average remaining LE between subjects with healthy weight and those who were overweight or obese. ${ }^{9}$ The same process was used to estimate the average QALYs lost and additional costs. The estimated average effects of YLL, QALYs and extra costs were scaled up to the entire 50-year-old population in each BMI category based on the 2015 projected Ghanaian population. ${ }^{25}$

\section{Model validity}

Validation of the model followed the recommendations of the International Society of Pharmacoeconomics and Outcomes Research Task-Force $7 .^{26}$ This was conducted in three ways: face validity, internal validity and external validity. Face validity was a subjective approach and involved people with health expertise in the disease area, to ensure the model incorporates the highest level of health and epidaemiological evidence. The overall structure of the model, population and outcomes were reviewed and validated by health economists (AJP, LS, SL), biostatisticians (LB, NM, GOB), a clinician (AJP) and an epidaemiologist (CGM). Internal validity using goodness of fit was performed to test whether the model correctly reproduced the input parameters. ${ }^{27}$ The model was used to reproduce sex-specific and age-specific mortalities and compared the sex-specific and age-specific mortalities used as input parameters. We fitted a linear regression and estimated the squared linear correlation coefficient $\left(\mathrm{R}^{2}\right)$, an index of the degree to which the data variation

Table 3 Key parameter in the model (direct healthcare costs (US\$))

\begin{tabular}{|c|c|c|c|c|}
\hline \multirow[b]{2}{*}{ Costs type } & \multicolumn{3}{|l|}{ BMI category } & \multirow[t]{2}{*}{ Distributions } \\
\hline & $\begin{array}{l}\text { Healthy weight } \\
\text { mean }(95 \% \mathrm{Cl})\end{array}$ & $\begin{array}{l}\text { Overweight } \\
\text { mean }(95 \% \mathrm{Cl})\end{array}$ & $\begin{array}{l}\text { Obese } \\
\text { mean }(95 \% \mathrm{Cl})\end{array}$ & \\
\hline OOP & 14.5 (10.3 to 18.6$)$ & 30.2 (18.9 to 41.5$)$ & 48.2 (25.6 to 70.8$)$ & Gamma \\
\hline NHIS & 20.3 (18.8 to 21.7 ) & 47.3 (41.7 to 53.0$)$ & 83.6 (60.7 to 96.40$)$ & Gamma \\
\hline
\end{tabular}

Direct healthcare costs (US $\$)^{17}$ were taken from our previous study.

NHIS, National Health Insurance Scheme; OOP, out of pocket. 
can be explained. As part of the internal validation process, one-way sensitivity analysis of key parameters was conducted to check whether the results changed in the direction expected when the base values were varied. By way of external validation, the model was used to predict life expectancies for males and females from age 25 to 85 years using a 5 -year age interval and compared with those in the life table. The estimated LEs were assumed to be valid if they fell within $1 \%$ of the observed LEs reported in the life tables when all the BMI categories are kept at their prevalence levels in the model.

\section{Uncertainty and sensitivity analyses}

Health economic models are mostly characterised by some degree of uncertainty. ${ }^{27}$ Tornado diagrams, oneway and probabilistic sensitivity analyses were used to address uncertainty. One-way sensitivity analyses were performed to identify the impact of key parameters on the outcomes by varying them from their base case values. The parameters were listed in tornado diagrams, ranked by the magnitude of their impact on the outcomes. The prevalence of healthy weight, overweight and obesity, the probability of death for all BMI categories, utility values and costs were varied by $\pm 20 \%$ of the values used in the base case analysis. ${ }^{27}$ Finally, probabilistic sensitivity analysis using second-order Monte Carlo simulation was conducted to incorporate multiple parameter uncertainties simultaneously. This allowed for the estimation of the CIs, which quantifies the level of uncertainties around the calculated LEs, QALYs and costs.

\section{Role of the funding source}

The SAGE Wave 2 was supported by WHO and the US National Institute on Aging's Division of Behavioural and Social Science Research through Interagency Agreements (OGHA 04034785; YA1323-08-CN-0020; Y1-AG1005-01) with WHO. Financial and in-kind support has come from the University of Ghana's Department of Community Health.

\section{Patient and public involvement}

This is a modelling study and did not directly involve patients and the public in the research question development, design or analysis. Rather the research development process was informed by the need for evidence to inform decision-making by policy-makers and health systems managers across the gatekeeping levels of healthcare that includes the community. A coauthor (SL) has worked at different gatekeeping levels and at the policymaking level in Ghana.

\section{RESULTS}

\section{Validity assessment}

Face validity was confirmed by our clinical, biostatistical, health economics and epidaemiological experts. The internal validation using the model to compare sexspecific and age-specific mortalities with published sexspecific and age-specific mortalities (online supplemental figure 1) obtained an $R^{2}$ of 0.999 for females and 0.998 for males. Univariate sensitivity analyses showed that the results changed in the expected direction when each input parameter was varied by $\pm 20 \%$. For example, when the base case probability of death for 50 -year-old women with obesity was varied by $\pm 20 \%$, LE varied from 23.7 to 24.5 years, QALYs ranged between 21.4 and 22.2 and costs from US $\$ 1131$ to US $\$ 1158$. For external validity, the overall hypothetical cohort in the model predicted LEs from age 25 years using a 5-year interval for males and females, which were compared and found to be almost identical to the LEs in the Ghanaian life table (online supplemental table 1 ). The $\mathrm{R}^{2}$ for the predictions and the published LEs were 0.998 in males and 0.999 in females.

\section{Model predictions for the base case (age $\mathbf{5 0}$ years)}

The results for the base case simulations are presented in table 4 for both males and females aged 50 years. The average remaining LE for healthy weight females was 25.6 (95\% CI: 25.4 to 25.8 ) years and healthy weight males was 23.0 (95\% CI: 22.8 to 23.2) years. LE of females and males who were overweight was 23.5 (95\% CI: 23.3 to 23.7) years and 20.7 (95\% CI: 20.5 to 20.9 ) years, respectively, and that for obese was 21.3 (95\% CI: 19.6 to 21.8) years and 17.6 (95\% CI: 17.5 to 17.8 ) years for females and males, respectively. The average remaining QALYs at age 50 years for those who were overweight (female: 21.0: 95\% CI: 20.8 to 21.2, male: 19.0: $95 \%$ CI: 18.8 to 19.2) and obese (female: 19.0: $95 \%$ CI: 18.8 to 19.7, male: 16.0: $95 \%$ CI: 15.8 to 16.2 ) were lower compared with those with healthy weight (female: 23.0: 95\% CI: 22.8 to 23.2, male: 21.0 : $95 \%$ CI: 20.8 to 21.2 ). The average total costs at the same age were higher for overweight (female: US\$1298: 95\% CI: 1290 to 1306, male: US $\$ 1177$ : 95\% CI: 1169 to 1186 ) and obesity (female: US\$2057: 95\% CI: 2043 to 2071, male: US $\$ 1831$ : 95\% CI: 1817 to 1846) compared with those with healthy weight (female: US\$619: $95 \%$ CI: 616 to 622 , male: US $\$ 571: 95 \%$ CI: 567 to 575$)$.

\section{YLL, QALYs losses and extra costs}

The base case analysis of average and population scaled up effect of overweight and obesity on LE, QALYs and healthcare costs are presented in table 5. The average effects for overweight were YLL (female: 2.1: 95\% CI: 1.9 to 2.3, male: 2.3: 95\% CI: 2.1 to 2.5), QALYs lost (female: 2.0: $95 \%$ CI: 1.8 to 2.3 , male: 2.0: $95 \%$ CI: 1.8 to 2.2 ) and extra total costs (female: US\$679: $95 \%$ CI: 675 to 683 , male: US\$607: 95\% CI: 602 to 611). The average effect due to obesity were YLL (female: $4.3: 95 \%$ CI: 4.1 to 4.5 , male: 5.4: $95 \%$ CI: 5.2 to 5.6), QALYs lost (female: 4.0: 95\% CI: 3.8 to 4.7 , male: 5.0: $95 \%$ CI: 4.8 to 5.2 ) and extra total costs (female: US\$1438: 95\% CI: 1427 to 1449 , male: US\$1260: 95\% CI: 1250 to 1271). When the average effect was scaled up to the total overweight population, notable losses were observed for YLL (females: 66 129: 95\% CI: 59771 to 72170 and males: 58 107: 95\% CI: 53348 to 62865 years), QALYs (females: 63 585: 95\% CI: 


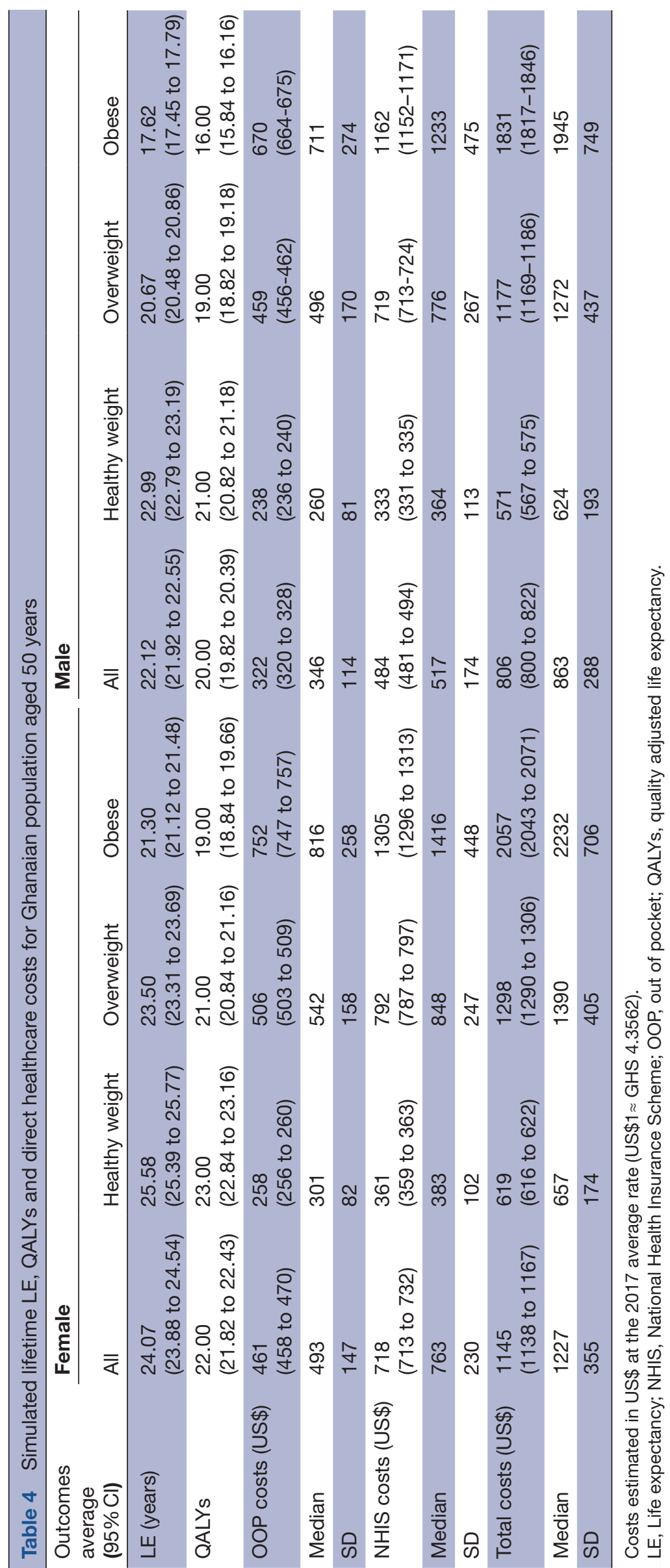




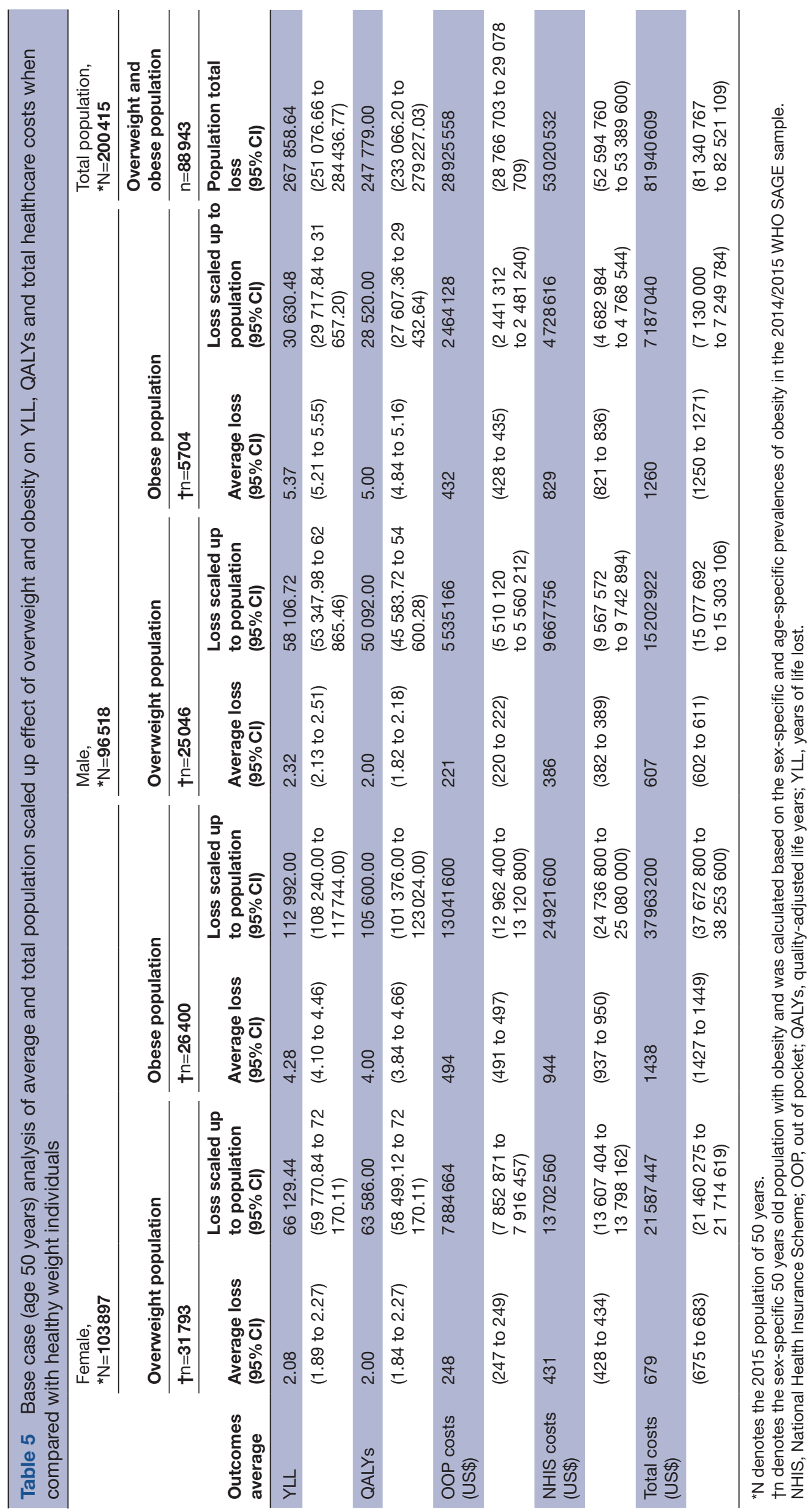


58499 to 72170 and males: 50 092: 95\% CI: 45584 to 54 600 years) and extra total costs (females: $\$ 21.5$ : $95 \% \mathrm{CI}$ : 21.4 to 21.7 million and males: US\$15.2: $95 \%$ CI: 15.0 to 15.3 million). For the population with obesity, substantial losses were estimated. The YLLs were 112992 (95\% CI: 108240 to 117744 ) and 30630 (95\% CI: 29718 to $31657)$ years in females and males, respectively. The QALYs lost were 105600 (95\% CI: 101376 to 123024) and 28520 (95\% CI: 27607 to 29433 ) years in females and males, respectively. Extra total costs were estimated at US $\$ 38.0$ (95\% CI: 37.6 to 38.2 ) million and US $\$ 7.1$ (95\% CI: 7.1 to 7.4 ) million in females and males, respectively. Of note, the health and economic burdens were substantially higher among females compared with males. Overweight and obesity combined contributed to 267869 (95\% CI: 251077 to 284437) YLL, 247779 (95\% CI: 233066 to 279227) lost QALYs and an extra cost of US $\$ 81.9$ (95\% CI: 81.3 to 82.5 ) million in the Ghanaian population aged 50 years.

\section{Sensitivity analysis}

One-way sensitivity analyses of key parameters showed impact on the LE, QALYS and costs for 50 year olds (online supplemental figures 2-4). It demonstrated that for LE, the most sensitive parameters were the probabilities of dying for healthy weight, overweight and for persons with obesity. For QALYs, the parameters with the greatest impact were HSUs for healthy weight, overweight and for persons with obesity. For costs, total costs for obesity, total costs for overweight and NHIS costs for obesity had the greatest impact. The probabilistic sensitivity analysis estimated the total impact of parameter uncertainty on the model and provided the CIs around the mean. It produced the best mean LEs, QALYs and costs. The CIs presented in table 4 and extrapolated to table 5 showed that the estimates were robust.

\section{DISCUSSION AND CONCLUSION}

The objective of this study was to quantify the long-term impact of overweight and obesity on LE, QALYs and total direct healthcare costs in Ghana. Our findings show that being overweight or obese is associated with significantly lower LEs and QALYs and higher healthcare costs, with these effects being higher in females. Overall, over a 50-year period, the aggregate losses due to overweight and obesity in the entire Ghanaian population aged 50 years were 267859 YLL, 247799 QALYs lost and an extra cost of US $\$ 82$ million, of which $64 \%$ will be borne by the government's NHIS.

Previous studies in other settings have shown that overweight and obesity are associated with YLL, loss of QALYs and increased costs. ${ }^{9} 1428$ For example, when the impact of obesity on QALYs and costs was examined in the USA over a 10 -year period, ${ }^{29}$ researchers found that being obese led to an average of 1.2 QALYs lost per person and increased total medical costs of US $\$ 42800$ per person compared with having healthy weight. Identical studies in the Belgian population ${ }^{28}$ modelled over a 20 -year period showed that a unit decrease in BMI resulted in improved QALYs and reduced costs. For example, Verhaeghe $e t a t^{28}$ found that in females, a 1-unit reduction in BMI among persons with obesity resulted in $€ 1039$ cost saving and in overweight, $€ 785$ was saved. They found that the cost impact was higher among females compared with males. Extrapolating this to the Belgian population resulted in an economic benefit of $€ 15.9$ million over the 20 -year period. The huge costs reported in these studies was because costs were estimated based on various obesityrelated diseases and disabilities, and the availability of national cost data.

In our study, overweight and obesity had dramatic adverse health and financial effects on individuals as well as the total population. Even though the average YLL was higher in males compared with females, the scaled up effect in the female population were higher mainly due to the higher prevalence of overweight and obesity in that population. Thus, in general, overweight and obesity have enormous impacts on the health and economic outcomes in the various studied populations, ${ }^{9} 1028$ and Ghana was no exception. In Ghana, the prevalence of overweight and obesity are steadily increasing, particularly in the female population. ${ }^{15}$ With looming crises of NCDs and an under-resourced health system with constant budget cuts, a huge impact of overweight and obesity on health and economic outcomes in the population necessitates urgent cost-effective interventions.

To our knowledge, this is the first study in Ghana and sub-Saharan Africa to have estimated the long-term effects of overweight and obesity on health and economic outcomes. $^{215}$ It is expected that most low-income and middle-income countries (LMICs) will likely face one of the biggest global health crises due to increasing rates of NCDs, yet healthcare is inequitable and inaccessible, and health facilities are under-resourced along with a limited proportion of the health budget available to manage NCDs. Besides the association with NCDs and higher all-cause mortality, ${ }^{20}$ obesity has been associated with reduced quality of life and LE, ${ }^{9}$ and high economic costs. ${ }^{528}$ Thus, for populations facing an increasing prevalence of overweight and obesity, the provision of concrete evidence of the long-term effects based on validated clinical and epidaemiological data, and the subsequent development of cost-effective interventions is vital. Our validated model has been developed at a very important time when decision-makers are confronted with challenges of identifying cost-effective measures to manage obesity as well as prioritisation of resources to achieve major local and global health goals. ${ }^{30}$

Cost-effectiveness analysis (CEA), particularly in NCDs, is generally lacking in most parts of Africa due to the lack of parameters, technical capacity and organisational support to do so. ${ }^{31}{ }^{32}$ To ensure the effective implementation of the Universal Healthcare Coverage and effective resource utilisation, Ghana has to proactively develop ways to reduce burden of 
disease. Hypertension, an NCD with one of its major risk factors as obesity, has been identified as one of the high spending and high burden disease areas ${ }^{33}$ and the Ministry of Health in Ghana commenced work in CEA of hypertension management. ${ }^{34}$ Effective management of such high burden disease areas requires evidencebased high-level resource prioritisation, and health systems, policies and operational preparedness. Thus, the model developed in this study becomes vital in the conduct of CEA to support resource prioritisation and preparedness specifically, in the area of NCDs. Our study has some limitations. First, the BMI-specific HRs used to calculate the BMI category-specific probability of dying in our model were taken from a large global population sample, ${ }^{20}$ and were not specific to Ghana. Second, in order to estimate the long-term outcomes, our model assumes that a subject would remain in the same BMI category over the period. Thus, the model overly depended on the current BMI prevalence rather than varying prevalence over time. As costs were estimated from self-reported health services utilisation, it might be subject to recall bias which may lead to overestimation or underestimation of the costs. Finally, costs used were limited to outpatient consultations and inpatient admissions ${ }^{17}$ due to the lack of other cost data, such as physiotherapy and dietician services and indirect costs (eg, cost associated with presenteeism and absenteeism from work) in the Ghanaian population. Thus, apart from the costs not reflecting the full direct healthcare costs in the population, we did not include indirect costs of overweight and obesity. However, studies have shown that obesity is increasingly associated with factors that reduce work productivity such as absenteeism and presenteeism, as well as short-term disabilities, ${ }^{29}$

Despite the above limitations in our study, most input parameters were estimated from the Ghanaian population for which the model has been developed, in-line with best practice recommendations..$^{35}$ Additionally, the internal and external validations and the sensitivity analyses performed showed that the clinical and epidaemiological data used in our model produced results that were close to the reality in this population. The choice of modelling technique through to reporting also followed best practice. ${ }^{13}$ Furthermore, the results from our modelling study bridge an important gap in the evidence in this area in Ghana and in most sub-Saharan African populations. Additionally, our model has been developed at a strategic time when most LMICs need to urgently develop sustainable and cost-effective weight reduction strategies to curb the burgeoning overweight and obesity prevalence. Some past initiatives that will require CEA in Ghana include the continual comprehensive behaviour change communication regarding obesity by the Ghana Health Services, ${ }^{36}$ and Ghana governments' strategic promotion of the use of locally produced healthy foods. ${ }^{37}$ The main idea behind these initiatives has been to support obesity prevention by highlighting the value of consuming 'whole, fresh and unprocessed ingredients'.
We have estimated the long-term impact of overweight and obesity on LE, YLL, QALYs and direct healthcare costs in Ghana. In conclusion, the study findings suggest that overweight and obesity are associated with substantial health and economic burdens in Ghana. This research provides essential evidence for policy-makers to set priorities during healthcare resource allocation. Our model can serve as a basis for future cost-effectiveness studies for weight management strategies for this population.

\section{Author affiliations}

${ }^{1}$ Menzies Institute for Medical Research, University of Tasmania, Hobart, Tasmania, Australia

${ }^{2}$ Department of Economics, University of Calgary, Calgary, Alberta, Canada ${ }^{3}$ Ministry of Health, Accra, GAR, Ghana

${ }^{4}$ The George Institute for Global Health, University of New South Wales, Sydney, New South Wales, Australia

${ }^{5}$ School of Health Policy \& Management, Nanjing Medical University, Nanjing, China ${ }^{6}$ Faculty of Medicine and Health, University of Sydney, Sydney, New South Wales, Australia

${ }^{7}$ Research Centre of Applied and Preventive Cardiovascular Medicine, University of Turku, Turku, Finland

${ }^{8}$ Public Health Program, Department of Kinesiology, College of Nursing and Health Innovations, University of Texas at Arlington, Arlington, Texas, USA

${ }^{9}$ National Research Council, Neuroscience Institute, Padova, Italy

${ }^{10}$ World Health Organization (WHO), Geneva, Switzerland

${ }^{11}$ Research Centre for Generational Health and Ageing, University of Newcastle, Newcastle, New South Wales, Australia

${ }^{12}$ Centre for Health Policy, School of Population and Global Health, The University of Melbourne, Melbourne, Victoria, Australia

\section{Twitter Stella Lartey @lartey_stella}

Acknowledgements We acknowledge and appreciate access to a preliminary version of Study on global AGEing and adult health (SAGE) Ghana Wave 2 data used for the analyses in this manuscript. We acknowledge the WHO SAGE Ghana team who implemented the surveys and the SAGE respondents.

Contributors SL was responsible for the formulation of the research idea, developed the analysis plan, conceptualised the paper, conducted the health economics analyses, wrote the manuscript and coordinated submission. LS and TL participated in the formulation of the research idea, study design, analysis, interpretation of data; validated the results and critically revised the manuscript. LB participated in the study design, analysis and interpretation of data, and critically revised the manuscript. AH, BdG, GOB and CGM participated in the study design and critically revised the manuscript. NM and PK participated in data acquisition and critically revised the manuscript. AJP participated in the formulation of the research idea, design of the study and study planning, participated in analysis and interpretation of the data, and critically revised the manuscript.

Funding AJP is funded by the Centre of Excellence in Population Ageing Research, Australian Research Council (CE170100005). The National Heart Foundation of Australia Future Leader Fellowship (100849) supports CGM. LS is supported by an National Health and Medical Research Council (NHMRC) Early Career Fellowship (grant number: GNT1139826). GOB is funded by the College of Nursing and Health Innovations. BdG is funded by the Menzies Community Fellowship. TL is currently supported by an NHMRC Early Career Fellowship (APP1141392) and National Heart Foundation Postdoctoral Fellowship (award ID 101956). The Study on global AGEing and adult health Wave 2 was supported by WHO and the US National Institute on Aging's Division of Behavioural and Social Science Research through Interagency Agreements (OGHA 04034785; YA1323-08-CN-0020; Y1-AG-1005-01) with WH0. Financial and in-kind support has come from the University of Ghana's Department of Community Health.

Competing interests None declared.

Patient and public involvement Patients and/or the public were not involved in the design, or conduct, or reporting, or dissemination plans of this research.

Patient consent for publication Not required. 
Ethics approval WHO SAGE was approved by the WHO Ethics Review Committee (reference number RPC149) with local approval from the University of Ghana Medical School Ethics and Protocol Review Committee (Ghana)

Provenance and peer review Not commissioned; externally peer reviewed.

Data availability statement Data are available upon request. Data from Study on global AGEing and adult health (SAGE) Ghana Wave 2 was used as the baseline data for this study. The necessary permission was obtained from WHO to use these data. Please visithttp://www.who.int/healthinfo/sage/cohorts/en/ for more details. The authors used the GhanaINDDataW2 and GhanaHHDataW2. The authors confirm that they had no special access privileges to the data. Interested researchers will have to submit a data request to WHO. Upon approval, the researchers will be granted access.

Open access This is an open access article distributed in accordance with the Creative Commons Attribution Non Commercial (CC BY-NC 4.0) license, which permits others to distribute, remix, adapt, build upon this work non-commercially, and license their derivative works on different terms, provided the original work is properly cited, appropriate credit is given, any changes made indicated, and the use is non-commercial. See: http://creativecommons.org/licenses/by-nc/4.0/.

\section{ORCID iDs}

Stella Lartey http://orcid.org/0000-0001-9519-7886

Lei Si http://orcid.org/0000-0002-3044-170X

\section{REFERENCES}

1 GBD 2015 Obesity Collaborators. Health effects of overweight and obesity in 195 countries over 25 years. N Engl J Med 2017;377:13-27.

2 Agyemang C, Boatemaa S, Frempong GA. Obesity in sub-Saharan Africa. In: Metabolic syndrome. Switzerland: Springer International Publishing, 2015: 1-13.

3 Buchmueller TC, Johar M. Obesity and health expenditures: evidence from Australia. Econ Hum Biol 2015;17:42-58.

4 Global Burden of Disease 2016 Disease and Injury Incidence and Prevalence Collaborators. Global, regional, and national incidence, prevalence, and years lived with disability for 328 diseases and injuries for 195 countries, 1990-2016: a systematic analysis for the global burden of disease study 2016. The Lancet 2018;390:1211-59.

5 Lehnert T, Sonntag D, Konnopka A, et al. Economic costs of overweight and obesity. Best Pract Res Clin Endocrinol Metab 2013;27:105-15.

6 Fryar C, Carroll M, Ogden C. Prevalence of overweight, obesity, and extreme obesity among adults aged 20 and over: United States, 1960-1962 through 2013-2014. Atlanta, GA National Center for Health Statistics, Surveys DoHaNE; 2016.

7 Finkelstein EA, Khavjou OA, Thompson $\mathrm{H}$, et al. Obesity and severe obesity forecasts through 2030. Am J Prev Med 2012;42:563-70.

8 Drummond MF, Sculpher MJ, Claxton K, et al. Methods for the economic evaluation of health care programmes. Fourth Edition. United Kingdom: Oxford University Press, 2015

9 Lung T, Jan S, Tan EJ, et al. Impact of overweight, obesity and severe obesity on life expectancy of Australian adults. Int $J$ Obes 2019;43:782-9.

10 Fontaine KR, Redden DT, Wang C, et al. Years of life lost due to obesity. JAMA 2003;289:187-93.

11 Finkelstein EA, Brown DS, Wrage LA, et al. Individual and aggregate years-of-life-lost associated with overweight and obesity. Obesity 2010;18:333-9.

12 Ministry of Health. Strategy for the management, prevention and control of chronic non-communicable diseases in Ghana 2012-2016. Ghana Ministry of Health; 2012.

13 Siebert U, Alagoz O, Bayoumi AM, et al. State-transition modeling: a report of the ISPOR-SMDM Modeling Good Research Practices Task Force--3. Value Health 2012;15:812-20.

14 Tucker DMD, Palmer AJ, Valentine WJ, et al. Counting the costs of overweight and obesity: modeling clinical and cost outcomes. Curr Med Res Opin 2006;22:575-86.
15 Lartey ST, Magnussen CG, Si L, et al. Rapidly increasing prevalence of overweight and obesity in older Ghanaian adults from 2007-2015: Evidence from WHO-SAGE Waves 1 \& 2. PLOS One 2019:14:1-16.

16 Lartey ST, Si L, de Graaff B, et al. Evaluation of the association between health state utilities and obesity in sub-Saharan Africa: evidence from World Health organization study on global ageing and adult health wave 2. Value Health 2019;22:1042-9.

17 Lartey ST, de Graaff B, Magnussen CG, et al. Health service utilization and direct healthcare costs associated with obesity in older adult population in Ghana. Health Policy Plan 2020;35:199-209.

18 Gray AM, Clarke PM, Wolstenholme JL, et al. Applied methods of cost-effectiveness analysis in health care. UK: Oxford University Press, 2012.

19 WHO. Life tables, 2018. Available: http://apps.who.int/gho/data/ view. main.60630?lang=en

20 Prospective Studies Collaboration, Whitlock G, Lewington S, et al. Body-Mass index and cause-specific mortality in 900000 adults: collaborative analyses of 57 prospective studies. Lancet 2009;373:1083-96.

21 Kowal P, Chatterji S, Naidoo N, et al. Data resource profile: the world Health organization study on global ageing and adult health (SAGE). Int J Epidemiol 2012;41:1639-49.

22 Subramanian SV, Özaltin E, Finlay JE. Height of nations: a socioeconomic analysis of cohort differences and patterns among women in 54 low- to middle-income countries. PLoS One 2011;6:e18962-13.

23 Cois A, Day C. Obesity trends and risk factors in the South African adult population. BMC Obesity 2015;2:1-10.

24 World Health Organization. Obesity: preventing and managing the global epidemic. Geneva: World Health Organization, 2000.

25 Ghana Statistical Service. 2010 population and housing census report: population projections/prospects. Ghana; 2014.

26 Eddy DM, Hollingworth W, Caro JJ, et al. Model transparency and validation: a report of the ISPOR-SMDM Modeling Good Research Practices Task Force--7. Value Health 2012;15:843-50.

27 Briggs $\mathrm{AH}$, Weinstein MC, Fenwick EAL, et al. Model parameter estimation and uncertainty: a report of the ISPOR-SMDM Modeling Good Research Practices Task Force--6. Value Health 2012;15:835-42.

28 Verhaeghe N, De Greve O, Annemans L. The potential health and economic effect of a body mass index decrease in the overweight and obese population in Belgium. Public Health 2016;134:26-33.

29 Su W, Huang J, Chen F, et al. Modeling the clinical and economic implications of obesity using microsimulation. J Med Econ 2015;18:886-97.

30 United Nations. Transforming our world: the 2030 agenda for sustainable development. New York United Nations; 2015.

31 Kularatna S, Whitty JA, Johnson NW, et al. Health state valuation in low- and middle-income countries: a systematic review of the literature. Value Health 2013;16:1091-9.

32 Panzer AD, Emerson JG, D'Cruz B, et al. Growth and capacity for cost-effectiveness analysis in Africa. Health Econ 2020;29:945-54.

33 de-Graft Aikins A, Kushitor M, Koram K, et al. Chronic noncommunicable diseases and the challenge of universal health coverage: insights from community-based cardiovascular disease research in urban poor communities in Accra, Ghana. BMC Public Health 2014;14 Suppl 2:S3.

34 Chalkidou K, Lord J, Gad M. Improving the quality and efficiency of healthcare services in Ghana through HTa: cost-effective care for managing hypertension in Ghana. London Minstry of Health Ghana and International Decision Support Initiative Imperial College London; 2017.

35 Drummond M, Barbieri M, Cook J, et al. Transferability of economic evaluations across jurisdictions: ISPOR good research practices Task force report. Value Health 2009;12:409-18.

36 Ministry of Health. National nutrition policy for Ghana 2013-2017. Ghana Ministry of Health; 2013.

37 Kumanyika S, Libman K, Garcia A. Wish obesity report 2013: strategic action to combat the obesity epidemic. Doha World Innovation Health Summit; 2013. 